\title{
Gamificação Aplicada à Educação: Uma Análise Bibliométrica Através do Método Webibliomining
}

\author{
Edgard da Cunha Pontes \\ Instituto Federal do Espírito Santo \\ edgardcunha@gmail.com \\ Márcio Tognere Gonçalves \\ Instituto Federal do Espírito Santo \\ marciotognere@gmail.com
}

\author{
Flávio Izo \\ Instituto Federal do Espírito Santo \\ fizo@ifes.edu.br \\ Lucas Vinco \\ Instituto Federal do Espírito Santo \\ lucasvinco@gmail.com
}

\begin{abstract}
RESUMO
Gamification has been widely cited as support for the student's school process. This growing interest has attracted the attention of researchers in the area. Therefore, a study using the Webibliomining method was conducted in order to map the scientific production related to gamification techniques applied in education. The data of this work were researched in the Scopus (Elsevier) database, considering the scientific production in the period from 2011 to 2020 . The methodological procedures used a descriptive and exploratory research, with a quantitative approach. The results showed 2.911 articles with the terms "Education AND Gamification". After a bibliometric analysis, it was found that the "peak" of production was in the year 2019 with 591 articles, which correspond to a difference of 137 records in relation to the previous year. The year 2020 was the year with the highest number of published articles, with 617 records. The USA was responsible for $13,60 \%$ of the total published articles. Computer Science was found to be the area with the most articles indexed (1.932), corresponding to 66,20\%. We sought to select articles to compose the "starting reference" in a bibliographic research on the theme "utilization of gamification in education".
\end{abstract}

\section{KEYWORDS}

Gamification, Education, Bibliometric Analysis, Webibliomining

\section{INTRODUÇÃO}

No cenário atual, a escola é desafiada a inovar, repensar e ressignificar todo o processo de ensino e aprendizagem. Para esta árdua tarefa, o uso de técnicas de gamificação na educação tem se mostrado bastante eficaz.

A definição de gamificação, segundo [4], é a utilização de mecânica, estética e pensamento baseados em games para engajar pessoas, motivar a ação, promover a aprendizagem e resolver problemas. De acordo com [6] e [9], um dos principais benefícios da gamificação é o aumento do engajamento do jogador.

Uma das necessidades mais importantes no cenário educacional atual, é a participação ativa do educando. Segundo [8], para atrair a atenção do aluno ao assunto estudado, convém estimular todos os sentidos. Desta forma, o uso de técnicas de gamificação aliados ao jogos educacionais digitais, demonstram um grande potencial para atender a esta demanda.

O autor [10] afirma que a tecnologia digital tem sido parte integrante da vida das crianças desde o seu nascimento. A essa geração ele denominou como "nativos digitais", já os pais e professores foram denominados como "imigrantes digitais". O uso de técnicas de gamificação na educação pode ser justamente a ponte entre estas duas gerações.

O objetivo principal deste artigo é o mapeamento da produção científica sobre a gamificação na educação, utilizando o método Webibliomining. O tema da pesquisa foi escolhido dada a relevância do número de publicações na área. Como resultado, foi desenvolvido um "núcleo de partida" com artigos selecionados na pesquisa, para servir de base para pesquisas futuras. Outros pesquisadores poderão utilizar este estudo para comparar os pontos abordados, além de expandir os limites e critérios aplicados neste trabalho.

O método Webibliomining pode ser definido como um conjunto inicial de referências bibliográficas através de métricas de documentos e informações, e ferramentas computacionais para uma investigação mais personalizada e específica sobre qualquer assunto de interesse científico/acadêmico, de acordo com [12].

O processo de pesquisa teve como foco os conceitos de gamificação e educação, e teve como objetivo responder às questões de pesquisa propostas.

Este trabalho está organizado como segue. Os principais conceitos são apresentados nas seções Seção 2 e Seção 3. Na Seção 4 são apresentados os resultados obtidos e as discussões sobre as Questões de Pesquisa. As considerações finais são apresentadas na Seção 5 .

\section{GAMIFICAÇÃO}

O primeiro uso documentado para o termo "gamification", ocorreu em 2008 (Deterding, Dixon, Khaled, \& Nacke 2011), segundo [7]. Gamificação, ou gamification em inglês, "consiste no processo de utilização de pensamento de jogos e dinâmica de jogos para engajar audiências e resolver problemas", segundo [15]. A designer de social games [5] define a gamificação como "a utilização de técnicas de games para tornar atividades mais divertidas e engajadoras”. Segundo a autora [1], nas duas definições apresentadas, podemos extrair o mesmo princípio, ou seja, ambas consideram a gamificação como o uso de elementos de jogos e técnicas de design de jogos em contextos diferentes de jogos. Abordando o contexto de aprendizagem, que é importante para este trabalho, o autor [4] define a gamificação como "a utilização de mecânica, estética e pensamento baseados em games para engajar pessoas, motivar a ação, promover a aprendizagem e resolver problemas".

Em [2], o autor sintetiza a gamificação como a utilização de recompensas para motivar pessoas, como os sistemas de fidelização 
de empresas aéreas e/ou restaurantes. Já a autora [11] define que gamificar é o mais sofisticado modo de educar. Ela ainda completa dizendo que é fundamental conscientizar os profissionais da educação da importância de entender como se processa o ensino e aprendizagem através da gamificação .

Para [14], a brincadeira pode ter papel fundamental no desenvolvimento da criança: "O jogo da criança não é uma recordação simples do vivido, mas sim a transformação criadora das impressões para a formação de uma nova realidade que responda às exigências e inclinações dela mesma”. Ou seja, o aprendizado também pode ser divertido.

\section{WEBIBLIOMINING}

Utilizou-se a metodologia webibliomining (ou mineração bibliográfica na rede web), proposta por [3], que pode ser incluída na interseção entre a Bibliometria, Bibliomineração, Informetria e Webometria, tendo por objetivo estabelecer um conjunto inicial de referências bibliográficas que permitirão o desenvolvimento de pesquisas em determinados temas, a partir de bases de dados disponíveis na rede web. Esta metodologia assemelhasse a uma revisão da literatura para identificar pontos importantes na literatura acadêmica sobre tema proposto no trabalho.

Esse modelo permite que o pesquisador consiga identificar os textos mais relevantes para iniciar uma pesquisa acadêmica, formando desta forma um "núcleo de partida", com base nas seguintes etapas:

(1) definição da amostra da pesquisa;

(2) pesquisa na amostra, com as palavras-chave;

(3) identificação dos periódicos com maior número de artigos publicados sobre o tema;

(4) identificação dos autores com o maior número de publicações;

(5) levantamento da cronologia da produção, identificando "ciclos de maior produção";

(6) seleção dos artigos para a composição do "núcleo de partida" para a pesquisa bibliográfica.

Desta forma, além da elaboração do núcleo de partida, este trabalho visa responder as seguintes Questões de Pesquisa (QPs).

QP1: Quais as principais contribuições do uso da gamificação na educação?

QP2: Quando se trata de gamificação inserida na educação, o Brasil tem acompanhado a evolução das publicações mundiais?

\section{RESULTADOS E DISCUSSÕES}

Nesta seção, são detalhadas cada uma das etapas do método Webibliomining.

\subsection{Definição da amostra}

A amostra pesquisada corresponde aos artigos indexados nas bases de dados ACM - Association for Computing Machinery, IEEE Xplore - Institute of Electrical and Electronics Engineers) e Scopus (Elsevier), com acesso pelo Portal de Periódicos Capes até Dezembro de 2020. A pesquisa nestas bases se deve ao fato da possibilidade de acesso a mesma, via o portal de periódicos e, principalmente, pela representatividade e abrangência. Quanto ao recorte temporal, a pesquisa foi realizada contemplando todos os anos que estavam disponíveis, tendo em vista que a primeira referência ao termo "gamification" surge no ano de 2011. A busca foi efetuada utilizando as palavraschave "gamification AND education" (utilizando aspas e o conector 'AND' para limitar os resultados).

Tabela 1: Distribuição de registros por base de dados

\begin{tabular}{lr}
\hline Base de Dados & Registros \\
\hline IEEE Xplore - Institute of Electrical and Electronics & 638 \\
Engineers) & \\
ACM - Association for Computing Machinery & 1.818 \\
Scopus (Elsevier) & 2.911 \\
\hline
\end{tabular}

Após analisar o total de registros e a abrangência das áreas de conhecimento dos principais registros, de acordo com a Tabela 1, todas as questões deste artigo são respondidas com base nos registros encontrados na base Scopus (Elsevier).

\subsection{Pesquisa na amostra}

Com base nos 2.911 registros retornados na base Scopus (Elsevier), cuja distribuição, por tipo de documento está apresentada no Tabela 2 .

Tabela 2: Distribuição de registros por tipo de documento

\begin{tabular}{lr}
\hline Tipo de Documento & Registros \\
\hline Conference Paper & 1.658 \\
Article & 896 \\
Conference Review & 134 \\
Book Chapter & 107 \\
Review & 78 \\
Book & 16 \\
Note & 10 \\
Editorial & 6 \\
Erratum & 3 \\
Data Paper & 2 \\
Letter & 1 \\
\hline
\end{tabular}

Os tipos de documentos mais encontrados foram: Conference Paper (1.654 registros) e Article (894 registros). Correspondendo a $56.94 \%$ e $30.77 \%$, respectivamente, do total de registros.

Continuando a análise do termo pesquisado, chegou-se a Tabela 3, que demonstra os países com mais de 75 publicações na área.

Apesar dos dados na Tabela 3 serem importantes, inicialmente preferiu-se não restringir as publicações por países. Para demonstrar a importância destes países na contribuição ao tema, os 13 países em voga, com 1979 registros que correspondem à $67.98 \%$ do total de publicações.

O destaque desta etapa do método, foram as contribuições dos EUA com 398 registros e Espanha com 340 registros, ocupando o primeiro e segundo lugar desta tabela, correspondendo a $13,60 \%$ e $10.92 \%$, respectivamente, do total de registros encontrados. O Brasil ocupa a $5^{\mathrm{a}}$ posição desta tabela, com 147 registros, que corresponde a $5.05 \%$ do total de publicações. 
Tabela 3: Distribuição de registros por país

\begin{tabular}{lr}
\hline País & Registros \\
\hline EUA & 398 \\
Espanha & 340 \\
Reino Unido & 190 \\
Alemanha & 176 \\
Brasil & 147 \\
Portugal & 112 \\
Austrália & 108 \\
Federação Russa & 94 \\
Malásia & 92 \\
Itália & 89 \\
Grécia & 79 \\
Canadá & 78 \\
Indonésia & 76 \\
\hline
\end{tabular}

\subsection{Identificação dos periódicos com maior número de artigos publicados}

Após a análise descobriu-se que existem 160 periódicos diferentes para as publicações encontradas. A Tabela 4 apresenta os dados referentes à distribuição de registros quanto ao título do periódico para aqueles que possuíam 30 registros ou mais. Essa amostra representa aproximadamente $26.80 \%$ do total de registros encontrados, com 780 registros.

Tabela 4: Distribuição de registros por veículo de publicação

\begin{tabular}{lr}
\hline Veículo de Publicação & Registros \\
\hline $\begin{array}{l}\text { Lecture Notes In Computer Science Including Sub- } \\
\text { series Lecture Notes In Artificial Intelligence And }\end{array}$ & 180 \\
$\begin{array}{l}\text { Lecture Notes In Bioinformatics } \\
\text { ACM International Conference Proceeding Series }\end{array}$ & 131 \\
Proceedings Of The European Conference On Games & 84 \\
Based Learning & 78 \\
$\begin{array}{l}\text { Ceur Workshop Proceedings } \\
\text { Communications In Computer And Information Sci- }\end{array}$ & 78 \\
ence & 68 \\
$\begin{array}{l}\text { Advances In Intelligent Systems And Computing } \\
\text { IEEE Global Engineering Education Conference }\end{array}$ & 62 \\
$\begin{array}{l}\text { Educon } \\
\text { ASEE Annual Conference And Exposition Confe- }\end{array}$ & 39 \\
rence Proceedings & \\
Proceedings Frontiers In Education Conference Fie & 30 \\
Proceedings Of The 11th European Conference On & 30 \\
Games Based Learning Ecgbl 2017 & \\
\hline
\end{tabular}

O destaque desta etapa do método, foram as publicações dos veículos Lecture Notes In Computer Science Including Subseries Lecture Notes In Artificial Intelligence And Lecture Notes In Bioinformatics e ACM International Conference Proceeding Series, cada um com 180 e 131 registros, correspondendo a $6.18 \%$ e $4.50 \%$ respectivamente, do total de registros encontrados.

\subsection{Identificação dos autores com maior número de publicações}

A Tabela 5 apresenta a distribuição de registros quanto à autoria das publicações. Esta tabela limita a apresentação dos autores que têm pelo menos 10 (dez) artigos publicados. A produção científica desses autores será rastreada e monitorada.

Tabela 5: Distribuição de registros por autores

\begin{tabular}{lr}
\hline Autor & Registros \\
\hline Isotani, S. & 20 \\
Bittencourt, I.I. & 15 \\
Antonaci, A. & 11 \\
Toda, A.M. & 11 \\
Barata, G. & 10 \\
Berkling, K. & 10 \\
Fonseca, D. & 10 \\
Gama, S. & 10 \\
Paiva, J.C. & 10 \\
Segura-Robles, A. & 10 \\
Villagrasa, S. & 10 \\
\hline
\end{tabular}

Os autores que mais contribuíram para ao tema da pesquisa foram: Isotani, S. (20 registros) e Bittencourt, I.I. (15 registros). Esses autores são importantes para identificar outras referências e contribuições futuras.

\subsection{Levantamento da cronologia da produção}

A Figura 1 apresenta os dados referentes à distribuição de registros quanto ao ano de publicação.

Através dos dados apresentados na Figura 1 pode-se observar que a produção científica no tema pesquisado tem evoluído, em uma escala cronológica. Analisando os dados referentes ao ano de publicação, observa-se que:

- os três artigos mais antigos indexados na base referem-se ao ano de 2011;

- é notável a curva de crescimento da produção científica deste tema, mantendo o ano posterior com maior número de produções até o ano de 2020.

- foi identificado que no ano de 2019, teve um pico de produção científica (137 registros com relação ao ano anterior).

- o Brasil vem acompanhando o crescimento do número de produção científica sobre o tema. Tendo 2019 como pico do número de registros.

\subsection{Resultados obtidos: identificação do "núcleo de partida"}

Conforme observado, foram encontradas inúmeras referências para os termos pesquisados "gamification" e "education". Ao todo, foram identificadas 2.911 publicações. Dessa forma, é notória a necessidade de um método para recorte das referências que devem ser utilizadas para pesquisas na área.

Segundo [3] sugere, o método Webibliomining é contemplando por quatro regras: 
Figura 1: Cronologia das Publicações

Todos os Países Brasil

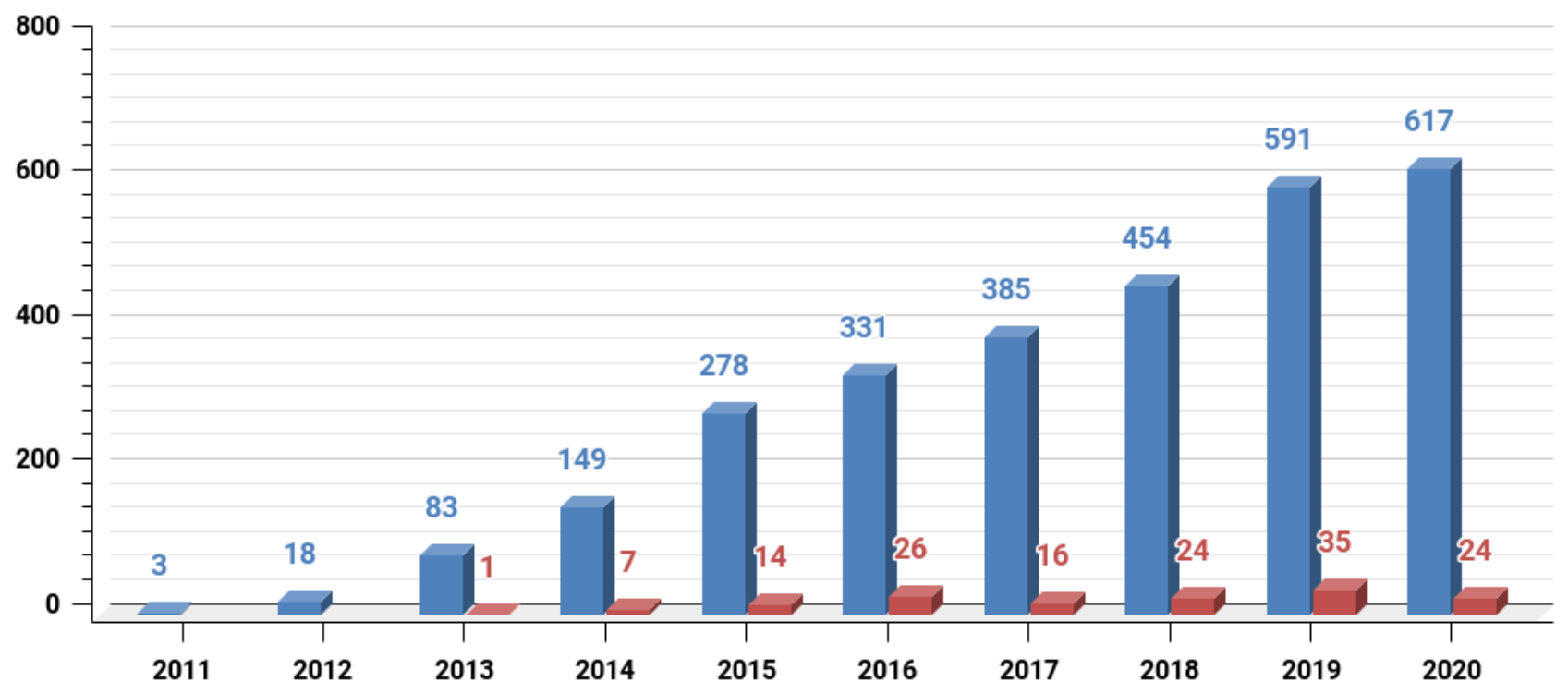

- Seleção dos 3 artigos mais antigos e de autores diferentes presentes na base. Esta regra busca identificar "linhas de pensamento diferentes" nas discussões iniciais (cadastradas na base). Assim, com base nesta regra foram selecionados os seguintes textos: HERBERT, Barry et al. (2011); COHEN, Aaron M. (2011); GRACE, Lindsay (2011).

- Seleção dos 10 artigos mais recentes e de autores diferentes presentes na base. Essa regra busca identificar "linhas de pensamento diferentes" nas discussões mais recentes (cadastradas na base). Através desta regra foram selecionados os registros dos seguintes autores: VEKUA, Natalya $\mathrm{N}$. et al (2020); FANENSHTIL, Tatiana V.; SADYKOVA, Irina V.; SUKHANOVA, Sofya Y. (2020); NESTERCHUK, Olga A.; GRISHIN, Oleg E.; CHEPURNAYA, Albina M. (2020); RADCHENKO, Galina; PERVUKHINA, Svetlana. (2020); ULMER, Jessica et al. (2020); CARRIÓN-TORO, Mayra et al. (2020); LEGAKI, Nikoletta-Zampeta et al. (2020); SMIDERLE, Rodrigo et al. (2020); SPANELLIS, Agnessa; DÖRFLER, Viktor; MACBRYDE, Jillian. (2020); BOVERMANN, Klaudia; BASTIAENS, Theo J. (2020).

- Seleção dos 10 artigos com maior grau de relevância, presentes na base. A própria Scopus fornece a opção de ordenação pelo grau de relevância. Com base nesta regra foram selecionados os registros dos seguintes autores: BICEN, Huseyin; AYDOGAN, Senay Kocakoyun. (2020); BROER, Jan. (2015); HOSHANG, Salam et al. (2018); KUSUMA, Gede Putra et al. (2018); NAH, Fiona Fui-Hoon et al. (2013); HAKAK, Saqib et al. (2019); GUI, Yuchen; ZHAO, Fan; HOYT, Eugene. (2019); RABAH, Jihan; CASSIDY, Robert; BEAUCHEMIN, Robert.
(2018); KENNY, Grace; LYONS, Roisin; LYNN, Theo. (2017); DEVERS, Christopher J.; GURUNG, Regan AR. (2015).

- Seleção de artigos com maior relevância para o ano com o "pico" de publicações (2019), identificado na seção 4.5. Com esta medida, busca-se identificar quais artigos tiveram maior relevância no momento de pico do tema pesquisado; ou seja: a evolução da moda. Que neste caso, é o ano anterior ao da pesquisa.

- 2019: artigos selecionados (7/591): GATTI, Lucia; ŠAŠINKA, Čeněk et al; EDWARDS, Bosede Iyiade et al; TODA, Armando M. et al; HURSEN, Cigdem; BAS, Cizem; ALMEIDA, Fernando; SIMOES, Jorge; VAN ROY, Rob; ZAMAN, Bieke.

A Tabela 6 apresenta a lista dos artigos selecionados para compor o "núcleo de partida" para a pesquisa. Esta tabela é o resultado da união dos resultados obtidos pela aplicação das regras do método Webibliomining, descritas anteriormente.

\subsection{QP1: Quais as principais contribuições do uso da gamificação na educação?}

De acordo com [13], a gamificação pode ser agrupada em quatro conceitos principais que explicam como aplicá-la no contexto educacional, são eles: Motivação (Motivation), Atitudes (Attitudes), Fluxo (Flow) e Aprendizagem (Learning).

Cada um desses conceitos podem ser explorados de forma dinâmica e personalizada, atendendo às peculiaridades da escola e/ou do público-alvo. 
Vale a pena ressaltar que este artigo não faz parte da Tabela 6 , por não atender aos critérios estabelecidos no método Webibliomining para esta pesquisa.

\subsection{QP2: Quando se trata de gamificação inserida na educação, o Brasil tem acompanhado a evolução das publicações mundiais?}

Foi possível analisar o crescimento do número de publicações no Brasil, e consequentemente da relevância ao termo abordado, de acordo com a Figura 1, que exibe o gráfico com a cronologia das publicações do Brasil e do total mundial.

O primeiro artigo de pesquisadores e/ou instituições brasileiras, foi identificado no ano de 2013. Com aumentos significativos nos anos de 2016 e 2019. O Brasil ocupa a $5^{a}$ posição na Tabela 3: Distribuição de registros por país, o que demonstra um interesse pelo tema de pesquisadores da área.

Os artigos a seguir não fazem parte do "núcleo de partida”, mas são importantes para pesquisas posteriores. Serão listados apenas os 5 artigos com o maior número de citações, encontrados na base de dados pesquisada:

- DE SOUSA BORGES, Simone et al. A systematic mapping on gamification applied to education. In: Proceedings of the 29th annual ACM symposium on applied computing 2014. p. 216-222.

- DA ROCHA SEIXAS, Luma; GOMES, Alex Sandro; DE MELO FILHO, Ivanildo José. Effectiveness of gamification in the engagement of students. Computers in Human Behavior, v. 58, p. 48-63, 2016.

- MAURÍCIO, R. de A. et al. A systematic mapping study on game-related methods for software engineering education. Information and software technology, v. 95, p. 201-218, 2018.

- PEDRO, Lais Z. et al. Does gamification work for boys and girls? An exploratory study with a virtual learning environment. In: Proceedings of the 30th annual ACM symposium on applied computing. 2015. p. 214-219.

- PAIVA, Ranilson et al. What do students do on-line? Modeling students' interactions to improve their learning experience. Computers in Human Behavior, v. 64, p. 769-781, 2016.

\section{CONSIDERAÇÕES FINAIS}

No período analisado neste trabalho, de 2011 a 2020, foi possível perceber um crescimento significativo do número de artigos indexados na base de dados Scopus (Elsevier) com os termos " gamification AND education”. Esse aumento no volume de artigos reflete justamente a importância e relevância das pesquisas nesta área. Os artigos do ano de 2021 foram desconsiderados para o "núcleo de partida".

Os pesquisadores dos Estados Unidos da América (EUA) publicaram 398 artigos, sendo o país com maior número de publicações, o que corresponde a $13,60 \%$ do total de artigos indexados. Foram identificados outros 12 países com maior número de publicações, sendo o Brasil o $5^{\circ}$ país com maior número de publicações, com 147 artigos. Na frente de países como Austrália (108 artigos), Portugal (112 artigos) e Itália (89 artigos).

A área de pesquisa que mais teve artigos publicados foi Computer Science com 1.932 artigos, o que equivale a $66,20 \%$ do total de artigos encontrados nesta pesquisa, seguida pela área Social Science, com 1.317 artigos publicados e 45,24\% do total de artigos.

As técnicas de gamificação tem se mostrado excelentes ferramentas para aumentar o engajamento de educandos no processo de aprendizagem.

A base de dados Scopus (Elsevier) atendeu aos objetivos propostos inicialmente, permitindo desta forma o desenvolvimento de um núcleo de partida apresentado neste trabalho, ampliando o conhecimento sobre a bibliografia da gamificação na educação, identificando os principais periódicos e autores da área, o que consequentemente, pode auxiliar outros trabalhos nesta área de pesquisa.

Outras bases de dados podem ser utilizadas em trabalhos futuros, assim como outros métodos de organização dos dados obtidos e critérios de seleção de artigos. Permitindo desta forma, a possibilidade de comparação dos resultados obtidos.

\section{REFERÊNCIAS}

[1] Flora Alves. 2015. Gamification: como criar experiências de aprendizagem engajadoras. DVS editora.

[2] Lynn Rosalina Gama Alves, Marcelle Rose da Silva Minho, and Marcelo Vera Cruz Diniz. 2014. Gamificação: diálogos com a educação. (2014).

[3] Helder Gomes Costa. 2010. Modelo para webibliomining: proposta e caso de aplicação. Revista da FAE 13, 1 (2010), 115-126.

[4] Karl M Kapp. 2012. The gamification of learning and instruction: game-based methods and strategies for training and education. John Wiley \& Sons.

[5] Amy Jo Kim. 2006. Community building on the web: Secret strategies for successful online communities. Peachpit press.

[6] Jane McGonigal. 2011. Reality is broken: Why games make us better and how they can change the world. Penguin.

[7] Craig Miller. 2013. The gamification of education. In Developments in Business Simulation and Experiential Learning: Proceedings of the Annual ABSEL conference, Vol. 40.

[8] Carolina Roberta Moraes and Simone Varela. 2007. Motivação do aluno durante o processo de ensino-aprendizagem. Revista eletrónica de Educação 1, 1 (2007), $1-15$.

[9] Cristina Ioana Muntean. 2011. Raising engagement in e-learning through gamification. In Proc. 6th international conference on virtual learning ICVL, Vol. 1. 323-329.

[10] Marc R Prensky. 2010. Teaching digital natives: Partnering for real learning. Corwin press.

[11] Tânia Maria Rodrigues da Rosa. 2018. Gamificação: uma prática para revitalizar a educação. (2018).

[12] Elindson Renney Alves Silva and Gisele Azevedo de Araújo Freitas. 2018. Uma Aplicação de Webibliomining para Pesquisas Científicas mais Eficientes. (2018).

[13] Rui Jorge Rodrigues da Silva, Ricardo Gouveia Rodrigues, and Carmem Teresa Pereira Leal. 2019. Gamification in management education: A systematic literature review. BAR-Brazilian Administration Review 16, 2 (2019).

[14] Lev Semenovich Vygotsky et al. 1988. Aprendizagem e desenvolvimento intelectual na idade escolar. Linguagem, desenvolvimento e aprendizagem 10 (1988), 103-117.

[15] Gabe Zichermann and Christopher Cunningham. 2011. Gamification by design: Implementing game mechanics in web and mobile apps. "O'Reilly Media, Inc.". 
Tabela 6: Artigos que compõem o Núcleo de Partida para a pesquisa (Acesso: 15/03/2021)

HERBERT, Barry et al. Dynamic Virtual Learning Landscapes to Enhance Student Reflective Processes. In: European Conference on Games Based Learning. Academic Conferences International Limited, 2011. p. 691.

COHEN, Aaron M. The gamification of education. The Futurist, v. 45, n. 5, p. 16, 2011.

GRACE, Lindsay. Gamifying archives, a study of docugames as a preservation medium. In: 2011 16th International Conference on Computer Games (CGAMES). IEEE, 2011. p. 172-176.

VEKUA, Natalya N. et al. Peculiarities of forming high-demanded soft skills in the educational space of the escape room. Perspectives of Science \& Education, n. 6, p. 397-412, 2020.

FANENSHTIL, Tatiana V.; SADYKOVA, Irina V.; SUKHANOVA, Sofya Y. Conceptualization of serious play in modern sociocultural reality. Perspectives of Science \& Education, n. 6, p. 31-39, 2020.

NESTERCHUK, Olga A.; GRISHIN, Oleg E.; CHEPURNAYA, Albina M. Digitalization as the "new normal" of higher education. In: Journal of Physics: Conference Series. IOP Publishing, 2020. p. 012068.

RADCHENKO, Galina; PERVUKHINA, Svetlana. Interactivity in digital teaching of a foreign language (on the example of Don State Technical University). In: E3S Web of Conferences. 2020. p. 18036.

ULMER, Jessica et al. Gamified Virtual Reality Training Environment for the Manufacturing Industry. In: 2020 19th International Conference on Mechatronics-Mechatronika (ME). IEEE, 2020. p. 1-6.

CARRIÓN-TORO, Mayra et al. iPlus a User-Centered Methodology for Serious Games Design. Applied Sciences, v. 10, n. 24, p. 9007, 2020. LEGAKI, Nikoletta-Zampeta et al. The effect of challenge-based gamification on learning: An experiment in the context of statistics education. International journal of human-computer studies, v. 144, p. 102496, 2020.

SMIDERLE, Rodrigo et al. The impact of gamification on students' learning, engagement and behavior based on their personality traits. Smart Learning Environments, v. 7, n. 1, p. 1-11, 2020.

SPANELLIS, Agnessa; DÖRFLER, Viktor; MACBRYDE, Jillian. Investigating the potential for using gamification to empower knowledge workers. Expert Systems with Applications, v. 160, p. 113694, 2020.

BOVERMANN, Klaudia; BASTIAENS, Theo J. Towards a motivational design? Connecting gamification user types and online learning activities. Research and Practice in Technology Enhanced Learning, v. 15, n. 1, p. 1, 2020.

BICEN, Huseyin; AYDOGAN, Senay Kocakoyun. Gamification Education for Parents: Effects on Motivation and Communication. Revista de Cercetare si Interventie Sociala, v. 69, p. 176, 2020.

BROER, Jan. Is This New? Family Resemblances in Gamification in Education. Bulletin of the Technical Committee on Learning Technology, v. 17, n. 4, p. 14-17, 2015.

HOSHANG, Salam et al. Factors influencing the adoption of education gamification within Abu Dhabi/UAE higher education institutions. In: Proceedings of the 10th International Conference on Education Technology and Computers. 2018. p. 145-151.

KUSUMA, Gede Putra et al. Analysis of gamification models in education using MDA framework. Procedia Computer Science, v. 135, p 385-392, 2018.

NAH, Fiona Fui-Hoon et al. Gamification of education using computer games. In: International Conference on Human Interface and the Management of Information. Springer, Berlin, Heidelberg, 2013. p. 99-107.

HAKAK, Saqib et al. Cloud-assisted gamification for education and learning-Recent advances and challenges. Computers \& Electrical Engineering, v. 74, p. 22-34, 2019.

GUI, Yuchen; ZHAO, Fan; HOYT, Eugene. Gamification in Mobile Application Development Education. In: International Conference on Human-Computer Interaction. Springer, Cham, 2019. p. 404-413.

RABAH, Jihan; CASSIDY, Robert; BEAUCHEMIN, Robert. Gamification in education: Real benefits or edutainment. In: Kidmore End: Academic Conferences International Limited. 2018. p. 489-496.

KENNY, Grace; LYONS, Roisin; LYNN, Theo. Don't make the player, make the game: exploring the potential of gamification in IS Education. 2017.

DEVERS, Christopher J.; GURUNG, Regan AR. Critical perspective on gamification in education. In: Gamification in education and business. Springer, Cham, 2015. p. 417-430.

GATTI, Lucia; ULRICH, Markus; SEELE, Peter. Education for sustainable development through business simulation games: An exploratory study of sustainability gamification and its effects on student'earning outcomes. Journal of cleaner production, v. 207, p. 667-678, 2019. ŠAŠINKA, Čeněk et al. Collaborative immersive virtual environments for education in geography. ISPRS International Journal of Geo-Information, v. 8, n. 1, p. 3, 2019.

EDWARDS, Bosede Iyiade et al. Haptic virtual reality and immersive learning for enhanced organic chemistry instruction. Virtual Reality, v. 23, n. 4, p. 363-373, 2019.

TODA, Armando M. et al. An approach for planning and deploying gamification concepts with social networks within educational contexts. International Journal of Information Management, v. 46, p. 294-303, 2019.

HURSEN, Cigdem; BAS, Cizem. Use of gamification applications in Science Education. International Journal of Emerging Technologies in Learning (iJET), v. 14, n. 01, p. 4-23, 2019.

ALMEIDA, Fernando; SIMOES, Jorge. The role of serious games, gamification and Industry 4.0 tools in the Education 4.0 paradigm. Contemporary Educational Technology, v. 10, n. 2, p. 120-136., 2019

VAN ROY, Rob; ZAMAN, Bieke. Unravelling the ambivalent motivational power of gamification: A basic psychological needs perspective. International Journal of Human-Computer Studies, v. 127, p. 38-50, 2019. 